\title{
Simulationsgestützte Konfiguration von piezoelektrischen Energy-Harvesting-Generatoren für die Versorgung von energieautarken Sensoren im Schienengüterverkehr und anderen Anwendungen
}

\author{
M. Koch ${ }^{1}$, W. Kaal' ${ }^{1}$, D. Mayer ${ }^{1}$ \\ ${ }^{1}$ Fraunhofer-Institut für Betriebsfestigkeit und Systemzuverlässigkeit LBF, Darmstadt, DE. \\ E-Mail: michael.koch@lbf.fraunhofer.de, william.kaal@lbf.fraunhofer.de, dirk.mayer@lbf.fraunhofer.de
}

\section{Zusammenfassung}

Energy Harvesting ermöglicht die Versorgung messtechnischer Systeme durch Wandlung der vor Ort vorhandenen, zumeist erneuerbaren Umgebungsenergie. Obwohl eine Vielzahl interessanter potentieller Anwendungen wie zum Beispiel autonome drahtlose Sensoren für den Schienengüterverkehr erforscht wurden, haben bisher nur wenige Energy-Harvesting-Systeme Marktreife erlangt. Ein Grund hierfür ist, dass die Energiebilanz eines durch Energy-Harvesting statt mit Batterien versorgten Systems in vielen Fällen nur schwierig vorhersagbar ist, was aber die Voraussetzung für die Beurteilung der Anwendbarkeit der Technologie in der Praxis darstellt. Bei der Auslegung zuverlässiger EnergyHarvesting-Systeme sollten daher die Charakteristika der Energiequelle und des Verbrauchers Berücksichtigung finden. Zur Steigerung der Marktrelevanz und Verwertbarkeit von Energy-HarvestingSensoren sind hierfür praxistaugliche Ansätze notwendig, die es ermöglichen, die Systeme einfach und anwendungsspezifisch zu konfigurieren. Diese Veröffentlichung stellt eine simulationsgestützte Methode vor, mit der sich vibrationsbasierte Energy-Harvesting-Sensoren auf verschiedene Befestigungspunkte mit stark schwankenden Vibrationseigenschaften konfigurieren lassen. Ziel ist die einfache Auswahl eines geeigneten Energiewandlers unter Berücksichtigung der realen Vibration, sodass eine festgelegte messtechnische Aufgabe energieautark und zuverlässig im späteren Betrieb abläuft. Die Umsetzung erfolgt mit einem leicht handhabbaren Werkzeug, um so den Einsatz von EnergyHarvesting-Sensoren in der industriellen Anwendung zu erleichtern.

Keywords: Energy-Harvesting, Smart-Sensor, Energieautarke Sensoren, piezoelektrischer Biegewandler

\section{Einleitung}

Micro-Energy-Harvesting hat sich bisher auf dem Markt nur in wenigen Anwendungen etabliert. Kommerzielle Produkte sind beispielsweise batterielose Schalter [1], die in der Gebäudeautomation eingesetzt werden. Die Systeme nutzen den manuellen Schaltvorgang als Energiequelle, um ein Schaltsignal energieautark an einen Empfänger, der in der Decke montiert ist, zu funken. Die Entwicklung von Energy-Harvesting-Applikationen erfordert einen ganzheitlichen Ansatz, da sich der energieautarke Betrieb nur erreichen lässt, wenn auch die Charakteristik der Energiequelle bei der Konfiguration berücksichtigt wird [2]. Bei batterielosen Schaltern bleibt die durch den Schaltvorgang generierte Energie konstant und lässt sich unabhängig vom Einsatzort charakterisieren. Möchte man aber beispielsweise die Vibrationen an einem Güterwagen [3], an einer Personenseilbahn oder an einem Antriebs- strang als Energiequelle nutzen, lässt sich der energieautarke Betrieb nur erreichen, wenn die durch Betriebs- und Umwelteinflüsse aber auch durch den Einsatzort bedingten Schwankungen bei der Konfiguration berücksichtigt werden. Die Anforderungen an die Auswahl des Vibrationsenergiewandlers steigen also mit der Komplexität der Charakteristik der verwendeten Energiequelle. Damit Energy-Harvesting gegenüber einer konventionellen Energieversorgung einen akzeptablen Zusatznutzen bietet, sind praxistaugliche Lösungen notwendig, die es ermöglichen, anwendungsspezifisch einen geeigneten Energiewandler zur Versorgung eines Smart-Sensors auszuwählen.

\section{Smart-Sensoren}

Energieautarke Sensoren haben keine drahtgebundenen Schnittstellen zum Datenaustausch mit ihrer Umgebung oder zur Energieversorgung. Da die verfügbare Energie be- 
grenzt ist, sind energieeffiziente Lösungen sinnvoll. Die klassische Messdatenerfassung und der direkte Austausch von Rohdaten sind in den meisten Fällen ineffizient. Die Effizienz lässt sich durch Smart-Sensoren verbessern, die die Messdatenerfassung und den Austausch mit einer Signalverarbeitung vereinen. Smarte-Sensoren bestehen meist aus LowCost-Komponenten (Abb. 1). Für die Erfassung von Beschleunigungsdaten lassen sich mikroelektromechanische Sensoren (MEMS) einsetzen. Für die Signalverarbeitung sind die Systeme mit Mikrocontrollern ausgestattet. Der Datenaustausch lässt sich über Low-PowerFunklösungen realisieren.

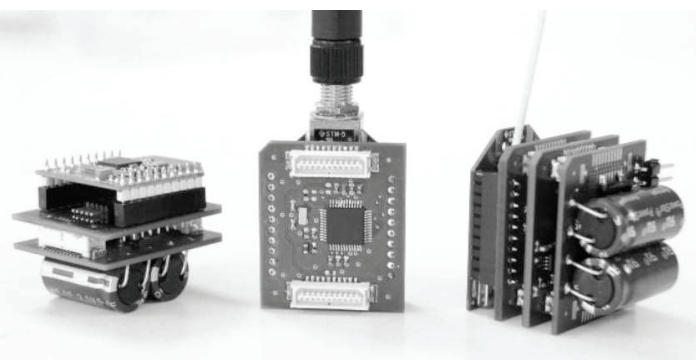

Abb. 1: Modular aufgebauter Smart-Sensor mit Low-Cost-Komponenten

Im BMBF-Projekt „Energieautarke Sensoren zur Zustandsüberwachung von Güterzügen (ESZüG)" [4], das im Rahmen des Förderschwerpunkts „Energieautarke Mobilität - Zuverlässige energieautarke Systeme für den mobilen Menschen" gefördert wurde, konnte zusammen mit einem Industriepartner [5] ein energieautarker Smart-Sensor zur Detektion von Flachstellen an Eisenbahngüterwagen entwickelt und im realen Betrieb erfolgreich getestet werden. Die Detektion erfolgt durch eine zum Radwinkel korrelierte Analyse der vertikalen Beschleunigungen am Achslagerdeckel des Radsatzes. Der Sensor bezieht die benötigte elektrische Energie aus den Vibrationen am Güterwagen und soll im Folgenden als Beispiel einer messtechnischen Anwendung dienen, für die ein geeigneter Energiewandler simulationsgestützt zu konfigurieren ist. Die für die Konfiguration benötigten Daten des energieautarken Flachstellendetektors sind in Tab. 1 zusammengefasst.

Tab. 1: Daten des im BMBF Projekt ESZüG entwickelten energieautarken SmartSensors zur Flachstellenerkennung an Eisenbahngüterwagen.

\begin{tabular}{|c|c|}
\hline Bezeichnung & Wert \\
\hline $\begin{array}{c}\text { Energiebedarf je } \\
\text { Messintervall }\end{array}$ & $20 \mathrm{~mJ}$ \\
\hline Messintervall & $10 \mathrm{~min}$ \\
\hline $\begin{array}{c}\text { Messbereich Zugge- } \\
\text { schwindigkeit }\end{array}$ & $30-100 \mathrm{~km} / \mathrm{h}$ \\
\hline
\end{tabular}

\section{Vibrationsenergiewandler}

Zur Energiewandlung lassen sich generell verschiedene Prinzipien und Bauformen von Wandlern nutzen. Das hier verwendete Prinzip nutzt den piezoelektrischen Effekt in $d_{31}$ Konfiguration in der Bauform eines fußpunkterregten bimorphen Biegewandlers (Abb. 2).

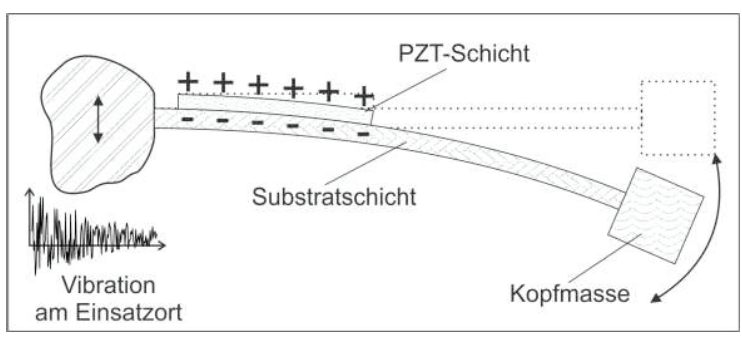

Abb. 2: Prinzip des piezoelektrischen Energy-Harvesting.

Der Wandler wird von der am Einsatzort vorhandenen Vibration am Fußpunkt angeregt. Aufgrund der Massenträgheit wird die Kopfmasse relativ zum eingeklemmten Fußpunkt ausgelenkt. Die höchste Wandlungseffizienz lässt sich im resonanten Betrieb erzielen, da dort die relative Auslenkung maximal ist. Bei Piezokeramiken (PZT) tritt wegen der molekularen Struktur grundsätzlich eine Verkopplung zwischen der mechanischen Dehnung und der elektrischen Ladung auf. Es kommt zu einer Ladungstrennung im Material. Aufgrund dieser Kopplung kann unter Zuhilfenahme einer Energy-Harvesting-Ladeschaltung [6][7] ein Teil der mechanischen Schwingungsenergie in elektrische Energie gewandelt und in einem Energiespeicher gespeichert werden.

\section{Hardware}

Hersteller von Energy-Harvesting-Generatoren bieten i.d.R. eine begrenzte Auswahl vorkonfigurierter Produkte an. Unter diesem Aspekt erfolgt die simulationsgestützte Konfiguration auf Basis von vier fest konfigurierten EnergyHarvesting-Generatoren entsprechend Abb. 3, die eine Produktreihe eines Herstellers [8] darstellen.

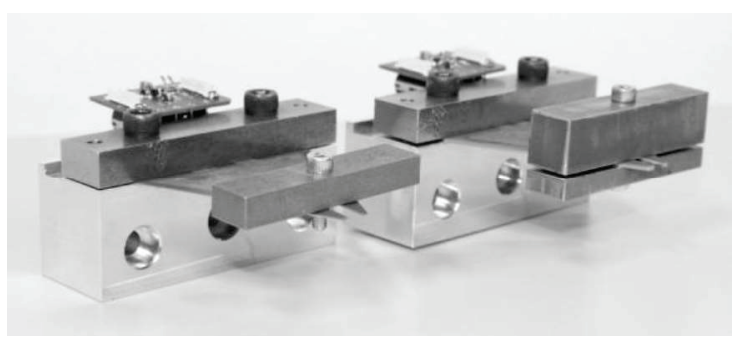

Abb. 3: Unterschiedlich konfigurierte piezoelektrische Energy-Harvesting Generatoren. Jeder Energy-Harvesting Generator bestehend aus den Komponenten piezoelektrischer Wandler, EH-Schaltung und Energiespeicher. 
Jeder Energy-Harvesting-Generator besteht aus einem piezoelektrischen Wandler, einer EH-Schaltung von Typ LTC3588 und einem Energiespeicher der Baureihe AVX BestCap. Dabei sind die Komponenten je Typ entsprechend Tab. 2 unterschiedlich konfiguriert.

Tab. 2: $\quad$ Daten der piezoelektrischen EnergyHarvesting-Generatoren der als Beispiel dienenden Produktreihe.

\begin{tabular}{|c|c|c|c|c|}
\hline Bezeichnung & Typ 1 & Typ 2 & Typ 3 & Typ 4 \\
\hline $\begin{array}{c}\text { Arbeitsfrequenz } \\
{[\mathrm{Hz}]}\end{array}$ & 50 & 50 & 75 & 100 \\
\hline $\begin{array}{c}\text { Abmessung } \\
{[\mathrm{cm}]}\end{array}$ & $6 \times 8 \times 2$ & $6 \times 8 \times 2$ & $6 \times 8 \times 1$ & $6 \times 8 \times 1$ \\
\hline $\begin{array}{c}\text { Energiespeicher } \\
{[\mathrm{mJ}]}\end{array}$ & 10 & 22 & 51 & 22 \\
\hline $\begin{array}{c}\text { Dicke Substrat } \\
{[\mathrm{mm}]}\end{array}$ & 0,7 & 0,7 & 0,7 & 0,2 \\
\hline $\begin{array}{c}\text { Dicke Piezo } \\
{[\mathrm{mm}]}\end{array}$ & 0,2 & 0,2 & 0,2 & 0,2 \\
\hline
\end{tabular}

\section{Simulationsgestützte Konfiguration von Energy-Harvesting-Sensoren}

Das mehrfache Testen unterschiedlicher Konfigurationen der Komponenten von EnergyHarvesting-Sensoren an der realen Anwendung ist meistens sehr aufwändig oder nicht möglich. Die Systeme müssen sich ohne großen Entwicklungs- und Testaufwand an die jeweilige Anwendung anpassen lassen und dort zuverlässig unter den realen, mitunter harten Betriebs- und Umweltbedingungen funktionieren. Daher wird hier auf eine simulationsgestützte Konfiguration zurückgegriffen. Ausgangspunkt ist dabei die Fragestellung, welcher Vibrationsenergiewandler aus einer Produktreihe (Abb. 3) geeignet ist, um einen bestimmten Smart-Sensor (Abb. 1) an einem neuen Einsatzort möglichst effizient mit der dort vorhandenen, aber schwankenden Vibrationsenergie zu betreiben. Bei der Simulation hat die umfassende Betrachtung aller Systemteile Vorrang vor dem Detaillierungsgrad der jeweiligen Ersatzmodelle. Zu den Systemteilen zählen die Charakterisierung der Energiequelle am Einsatzort, die Betrachtung des Wandlungs- und Speicherungsverhaltens der Vibrationsenergiewandler und die Berücksichtigung des Energiebedarfs des Smart-Sensors.

\section{Energiequelle}

Für die Konfiguration eines Energy-HarvestingSensors ist die Kenntnis darüber, wann und in welcher Menge Energie generiert werden kann, zwingend notwendig. Andernfalls ist eine Skalierung zwischen Smart-Sensor und Energiewandler nicht möglich. Jede Energiequelle lässt sich neben ihrer Energieform und ihrem Wandlungsprinzip anhand ihrer Energieverfügbarkeit charakterisieren. Schon die Gegen- überstellung der Energieformen Wind, Wasser und Solar zeigt, wie unterschiedlich die Charakteristik der Verfügbarkeit sein kann. Auch die Anwendung nimmt sehr stark Einfluss auf die Verfügbarkeit der Energiequelle. Die Charakteristik einer auf dem Solarprinzip arbeitenden Energiequelle besitzt im Innenraum eines Gebäudes mit künstlichem Licht eine vollkommen andere Dynamik als im Außenbereich. Gerade bei der Nutzung von mechanischer Vibrationsenergie wird die Charakteristik der Energiequelle besonders durch die Anwendung und den Einsatzort bestimmt. Für die Konfiguration muss dieser Sachverhalt in einem beherrschbaren Rahmen genügend genau vorhersagbar sein. Die Auslegung ist nur dann sinnvoll möglich, wenn die Charakteristik anhand eines Ersatzmodells mit vertretbarem Aufwand beschreibbar ist [9].

\section{Betriebsdatenerfassung}

Das Verhalten einer Vibrationsquelle lässt sich im Allgemeinen mit Beschleunigungssensoren messen. In vielen Anwendungen lassen sich hierfür auf dem Markt erhältliche Low-CostDatenlogger einsetzen. In Abhängigkeit der Komplexität der Energiequelle ist es im Rahmen der Charakterisierung sinnvoll, die Vibrationszeitreihen anhand von auftretenden Betriebs- und Umweltzuständen zu klassifizieren. Hierzu sollten neben der Beschleunigung weitere Systemgrößen gemessen werden.

\section{Charakterisierung}

Eine systematische Kategorisierung und Analyse der Messdaten bildet die Basis für die Charakterisierung der Energiequelle. Abb. 4 zeigt auszugsweise einige Ergebnisse einer Charakterisierung aus dem Schienengüterverkehr [3]. Energiequelle ist die im regulären Bahnbetrieb auftretende vertikale Vibration am Lagerkasten eines Y25-Drehgestells, das an einem Kesselwagen vom Typ Zarcens $75 \mathrm{~m}^{3}$ verbaut ist. Der Smart-Sensor zur Detektion von Flachstellen wird hier montiert. Im Rahmen der Charakterisierung der Energiequelle wurden die im Bahnbetrieb gemessene Vertikalbeschleunigung anhand von Betriebszuständen gemäß der Beladung und der Zuggeschwindigkeit analysiert. In Abb. 4 sind die gemittelten Frequenzspektren von sechs unterschiedlichen Betriebszuständen abgebildet. Die drei Diagramme beziehen sich auf die Zuggeschwindigkeiten $80 \mathrm{~km} / \mathrm{h}, 90 \mathrm{~km} / \mathrm{h}$ und $100 \mathrm{~km} / \mathrm{h}$. Die beiden Spektren je Diagramm beschreiben das mittlere spektrale Vibrationsverhalten des Generator-Fußpunkts bei gleichbleibender Zuggeschwindigkeit für den unbeladenen und den beladenen Zustand. Der Vergleich der Spektren zeigt, dass einige Peaks ihre Frequenz in Abhängigkeit der Zuggeschwindigkeit ändern (siehe Markierung 1). 


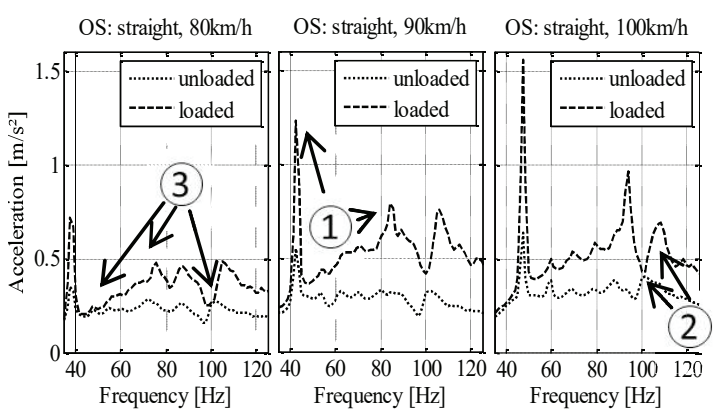

Abb. 4: Mittelwert der Frequenzspektren ausgewählter Betriebszustände. (1) Geschwindigkeitsabhängige Peaks. (2) Beladungsabhängiger Peak. (3) Arbeitsfrequenzen der EnergyHarvesting-Generatoren.

Diese Frequenzen werden periodisch durch die regelmäßigen Abstände der Eisenbahnschwellen angeregt. Es ist auch zu erkennen, dass die Spektralamplituden im beladenen Zustand im Allgemeinen höher sind als im unbeladenen. Es lässt sich auch beobachten, dass sich ein Peak bei $106 \mathrm{~Hz}$ im beladenen Zustand auf $101 \mathrm{~Hz}$ im unbeladenen Zustand verschiebt (siehe Markierung 2).

Bei der Konfiguration des Energy-HarvestingSensors stellt sich die Frage, welcher EnergyHarvesting-Generator aus der Produktreihe (Tab. 2) für die Versorgung des Smart-Sensors entsprechend den Daten in Tab. 1 geeignet ist. Dabei muss garantiert werden, dass für Zuggeschwindigkeiten zwischen $30 \mathrm{~km} / \mathrm{h}$ und 100 $\mathrm{km} / \mathrm{h}$ der je Messintervall benötigte Energiebedarf von $20 \mathrm{~mJ}$ innerhalb von 10 min durch den Energy-Harvesting-Generator zur Verfügung gestellt wird. Hierzu wurden repräsentative Zeitreihen aus den Messdaten extrahiert und in der Simulation verwendet.

\section{Piezoelektrischer Biegewandler}

Für eine vereinfachte Berechnung der generierten Leistung genügt in der Regel ein parametrisches Modell des Generators und der elektrischen Last. Unter Ausnutzung der elektromechanischen Analogie können die mechanischen Elemente als elektrische Komponenten abgebildet und so einer gekoppelten Simulation leicht zugänglich gemacht werden.

Der mechanische Schwinger wird so durch den Schwingkreis bestehend aus $m_{m}, c_{m}$ und $d_{m}$ abgebildet (Abb. 5). Die elektromechanische Kopplung wird durch den dimensionsbehafteten Transformator mit dem Übersetzungsverhältnis $\alpha$ dargestellt. Die Anregung erfolgt über eine ideale Stromquelle, deren Stromstärke der Fußpunktgeschwindigkeit des Generators entspricht. Mit Hilfe üblicher Beziehungen für elektrische Netzwerke ergibt sich die in Gleichung 1 dargestellte Übertragungsfunktion $V$ von der Fußpunktbeschleunigung zur Generatorspannung bei vernachlässigbarem Wider- stand $R_{p}$ und ohne elektrische Last $Z_{\text {Load }}$ (Leerlaufbetrieb).

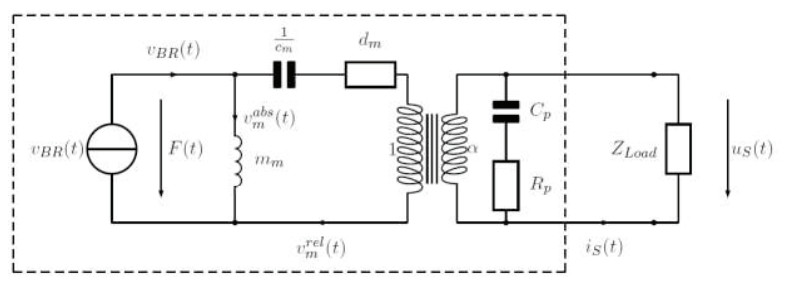

Abb. 5: Die Abbildung zeigt das in der Simulation zugrunde gelegte modale Modell des fußpunkterregten piezoelektrischen Biegewandlers als elektrischen Kreis.

$$
V=\frac{U_{S, 0}(t)}{\dot{v}_{B R}(t)}=\frac{m_{m}}{\frac{\alpha^{2}}{C_{P}}+c_{m}+d_{m} s+m_{m} s^{2}} \frac{\alpha}{C_{P}}
$$

Die einzelnen Modellparameter können direkt aus den konstruktiven Kenngrößen des Generators ermittelt bzw. aus Messungen bestimmt werden. Dabei werden nichtlineare Anteile im Materialverhalten vernachlässigt. Die modale Masse $m_{\mathrm{m}}$ der ersten Biegeeigenform entspricht in guter Näherung der schwingenden Endmasse, die Resonanzfrequenz und die Piezokapazität können direkt gemessen werden. Steifigkeit, Dämpfung und Wandlerkonstante lassen sich durch indirekte Identifikation schätzen (Abb. 6).

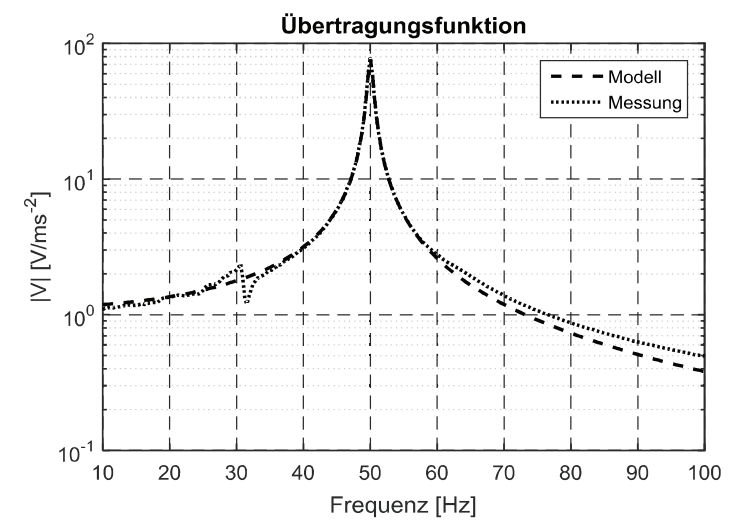

Abb. 6: Indirekte Identifikation der Modellparameter des piezoelektrischen Biegewandlers. Die Parameteranpassung erfolgt mit Hilfe der Methode der gewichteten kleinsten Fehlerquadrate.

Hierbei werden die fehlenden Parameter Steifigkeit, Dämpfung und Wandlerkonstante durch Annäherung der Funktion $V$ an die gemessenen Übertragungsfunktion $V_{\text {mes }}$ angepasst. Die Parameteranpassung lässt sich mit Hilfe der Methode der gewichteten kleinsten Fehler- 
quadrate automatisieren. In Tabelle 3 sind die Modellparameter für die Energy-HarvestingGeneratoren von Typ 1 und 2 zusammengefasst.

Tab. 3: Gemessene und berechnete Modellparameter der piezoelektrischen Energy-Harvester von Typ 1, 2 (vergleiche Tab. 2).

\begin{tabular}{|c|c|c|c|}
\hline \multicolumn{2}{|l|}{ Bezeichnung } & \multicolumn{2}{|c|}{ Gemessen } \\
\hline Masse & $m_{m}$ & 0,15 & $\mathrm{~kg}$ \\
\hline Resonanzfrequenz & $f_{\text {ges }}$ & 50,03 & $\mathrm{~Hz}$ \\
\hline Piezokapazität & $C_{P}$ & 55 & $\mathrm{nF}$ \\
\hline \multicolumn{2}{|l|}{ Bezeichnung } & \multicolumn{2}{|c|}{ Geschätzt } \\
\hline Steifigkeit & $c_{m}$ & 14119 & $\mathrm{~N} / \mathrm{m}$ \\
\hline Dämpfung & $d_{m}$ & 0,6838 & $\mathrm{Ns} / \mathrm{m}$ \\
\hline Wandlerkonstante & $\alpha$ & 0,0062 & $\mathrm{As} / \mathrm{m}$ \\
\hline
\end{tabular}

\section{Energy-Harvesting-Schaltung und Energie-} speicher

Die Modellbildung der elektrischen Last $Z_{\text {Load }}$ (siehe Abb. 5) richtet sich nach dem Verhalten der verwendeten EH-Ladeschaltung vom Typ LTC3588 der Firma Linear Technology und dem Energiespeicher $\mathrm{C}_{\mathrm{ES}}$ der Baureihe AVX BestCap Pulse Superkondensatoren. Der IC LTC3588 kombiniert einen VollBrückengleichrichter mit einem Abwärtswandler (Buck Regulator) auf einem Chip und eignet sich für piezoelektrische Anwendungen (Abb. 7).

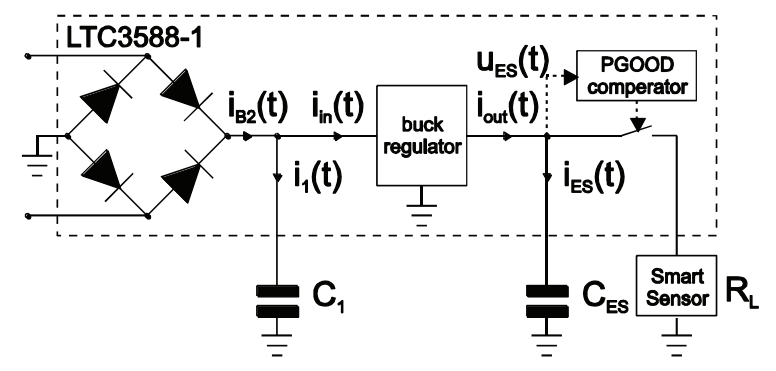

Abb. 7: Schematische Darstellung der elektrischen Last $Z_{\text {Load }}$ bestehend aus einem Brückengleichrichter mit Glättungskondensator, einem $A b$ wärtswandler, einem Energiespeicher und einem Smart-Sensor.

Die jeweiligen Komponenten der elektrischen Last $Z_{\text {Load }}$ lassen sich durch einfache Ersatzmodelle in die gekoppelte Simulation einbinden. Der Abwärtswandler lässt sich vereinfacht mit Hilfe eines Zweipunkreglers mit Hysterese in einem Modell beschreiben (Abb. 8). Der Energietransfer erfolgt in Abhängigkeit der Ladespannung des Glättungskondensator $C_{1}$ und wird Ausgangsseitig im Energiespeicher $\mathrm{C}_{\mathrm{ES}}$ gespeichert.

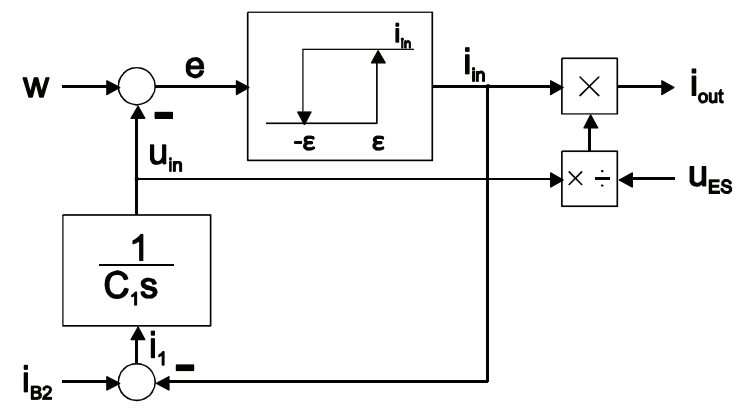

Abb. 8: Modellbildung für den Abwärtswandler (Buck Regulator) des LTC 3588-1 durch einen Zweipunkregler mit Hysterese.

In Abhängigkeit der Ladespannung $\mathrm{u}_{\mathrm{ES}}$ des Energiespeichers schaltet ein Komparator den Smart-Sensor ein, der vereinfacht durch eine entsprechend dem Messintervall geschaltete ohmsche Last $R_{L}$ abgebildet wird. Das Ersatzmodell des Komparators lässt sich ebenfalls durch einen Zweipunktregler mit Hysterese entsprechend Abb. 9 in der Simulation berücksichtigen.

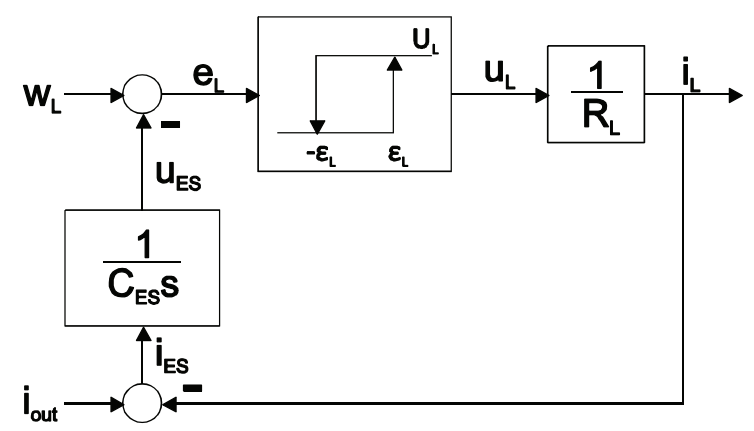

Abb. 9: Modellbildung des PGOODKomparators des LTC 3588-1 durch einem Zweipunktregler mit Hysterese.

Die Kapazität des Energiespeichers wird entsprechend der in der Baureihe AVX BestCab vorgegebenen Werte im Simulationsmodell parametriert (Tab. 4).

Tab. 4: Maximale verfügbare Energiemenge je Messintervall in Abhängigkeit vorgegebenen Kapazitäten der Produktreihen der Energiespeicher.

\begin{tabular}{|c|c|c|}
\hline $\begin{array}{c}\text { Typ } \\
\text { (Tab. 2) }\end{array}$ & $\begin{array}{c}\text { Kapazität } \\
\text { [mF] }\end{array}$ & $\begin{array}{c}\text { Speicherbare } \\
\text { Energie [mJ] }\end{array}$ \\
\hline 1 & 10 & 10,4 \\
\hline 2,4 & 22 & 22,8 \\
\hline 3 & 50 & 51,8 \\
\hline
\end{tabular}

Die Parameteranpassung von $R_{L}$ erfolgt entsprechend des Energiebedarfs des SmartSensors (hier $20 \mathrm{~mJ}$ je Messintervall, vergleiche Tab.1). 


\section{Simulationsanwendung und Konfiguration}

Die Modelle der einzelnen Komponenten wurden in einer Simulation in Simulink SimScape gekoppelt. Für jeden der in Tab. 2 beschriebenen Energy-Harvesting-Generatoren wurden je ein Modell entsprechend der gemessenen und berechneten Modellparameter angepasst. Die parametrierten Modelle wurden mit dem Matlab Compiler in eine ausführbare Anwendung übersetzt und in eine weitere eigenständige Anwendung mit grafischer Benutzeroberfläche (GUI) eingebunden (Abb. 10).

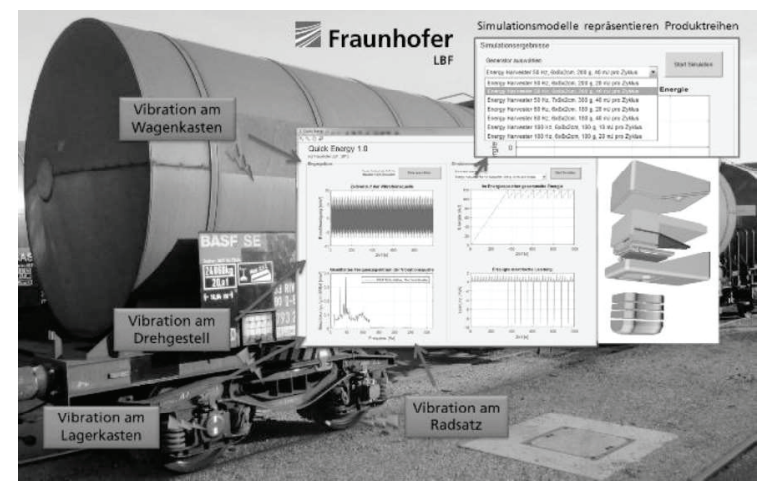

Abb. 10: Quick Energy ist eine simulationsgestütztes Werkzeug zur Auswahl eines geeigneten Energy-HarvestingGenerators aus eine Produktreihe.

Die Produktreihe lässt sich flexibel erweitern. Über die GUI können die Zeitreihen der Messdaten, die die Charakteristik der Energiequelle am Einsatzort repräsentieren, eingelesen und spektral analysiert werden. Ein Menü ermöglicht die Auswahl eines Energy-HarvestingGenerators der Produktreihe. Anhand der eingelesenen Messdaten konnte die Energiebilanz des Energy-Harvesting-Sensors für den jeweils ausgewählten Energiewandler simuliert werden. Dadurch war es möglich, unter Berücksichtigung der realen Vibrationsdaten am spezifischen Einsatzort und der Anforderungen seitens des Smart-Sensors das Gesamtsystem zu konfigurieren. Abb. 11 zeigt den simulierten Verlauf der Ladespannung des Energiespeichers bei Versorgung des Smart-Sensors aus Tab. 1 für den Energy-Harvesting-Generator Typ 2 aus Tab. 2. Im Labortest wurden die im realen Bahnbetrieb gemessenen Vibrationen für die verschiedenen Betriebszustände auf einem Vibrationsshaker nachgestellt. Zur Validierung der Simulationsmodelle wurden diese Beschleunigungsdaten in die Simulationsanwendung eingelesen und die gleiche Konfiguration simuliert. Es zeigte sich, dass der Energy-Harvesting-Generator vom Typ 2 für die Versorgung geeignet ist. Dieser wurde auch im Rahmen des Testbetriebs am realen Güterwagen eingesetzt. Typ 4 ist nicht geeignet, um die

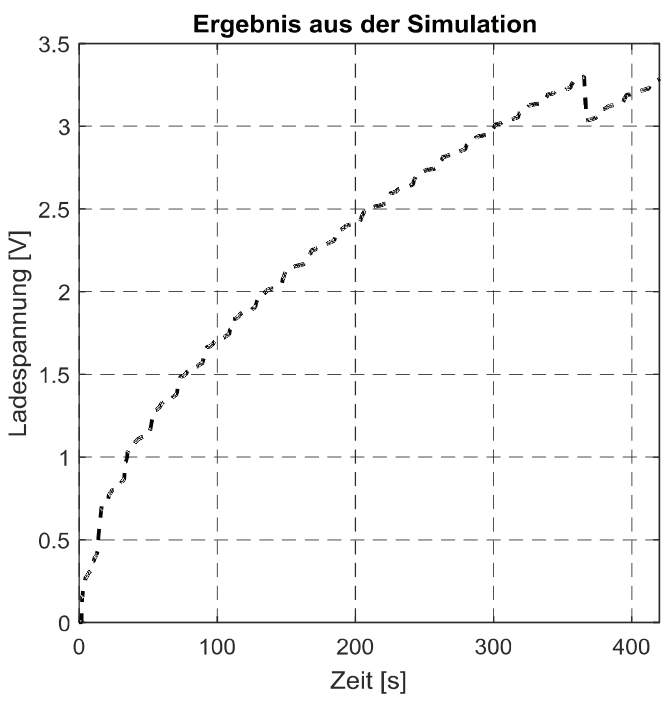

Abb. 11: Simulationsergebnis bei realer Vibration im Bahnbetrieb im Fall einer Zuggeschwindigkeit von $100 \mathrm{~km} / \mathrm{h}$. Das Diagramm zeigt den simulierten Verlauf der Ladespannung des Energiespeichers.

benötigten $20 \mathrm{~mJ}$ in einem Zeitraum von 10 min zu generieren. Grund dafür ist, dass die reale Vibrationsamplitude bei bestimmten Betriebszuständen zu gering wird. Hauptursache ist, dass der piezoelektrische Biegewandler von Typ 4 aufgrund seiner Geometrie eine im Verhältnis zur Anregung zu geringe Resonanzüberhöhung $V\left(f_{\text {res }}\right)$ aufweist. Dadurch ist die gleichgerichtete Spannung im Glättungskondensator $C_{1}$ (Abb. 7) zu klein, sodass die obere Hysterese-Schwelle des Abwärtswandlers (Abb. 8) vielfach nicht erreicht wird. Durch diesen nichtlinearen Effekt war der energieautarke Betrieb des Smart-Sensors bei besonders geringen Zuggeschwindigkeiten nicht möglich. Dies wurde durch Laboruntersuchungen bestätigt. Der Energy-HarvestingGenerator Typ 1 scheidet aufgrund des zu kleinen Energiespeichers von $10 \mathrm{~mJ}$ aus. Der energieautarke Betrieb beim Einsatz des Energy-Harvesting-Generators Typ 3 ist prinzipiell auch möglich. Der zu große Energiespeicher führt jedoch zu einem ineffizienten Betrieb. Hat sich der Energiespeicher nach langen Standzeiten entladen, benötigt Typ 3 länger Ladezeiten bis es einsatzbereit ist als Typ 2. Des Weiteren ist die mechanische Belastung bei Typ 3 mit einer Arbeitsfrequenz bei 75 $\mathrm{Hz}$ in bestimmten Betriebszuständen im Geschwindigkeitsbereich zwischen $30 \mathrm{~km} / \mathrm{h}$ und $100 \mathrm{~km} / \mathrm{h}$ aufgrund der geschwindigkeitsabhängigen Peaks höher als bei der Arbeitsfrequenz $50 \mathrm{~Hz}$ (Abb. 4). In Tab. 5 sind die wesentlichen Ergebnisse aus der simulationsgestützten Konfiguration zusammengefasst. 
Tab. 5: Zusammenfassung der wesentlichen Ergebnisse aus der simulationsgestützten Konfiguration

\begin{tabular}{|c|c|c|}
\hline $\begin{array}{c}\text { Typ } \\
\text { (Tab. 2) }\end{array}$ & Geeignet & Beschreibung \\
\hline 1 & Nein & $\begin{array}{c}\text { Zu kleiner Energie- } \\
\text { speicher }\end{array}$ \\
\hline 2 & Ja & $\begin{array}{c}\text { Liefert 20 mJ im } \\
\text { Messintervall von 10 } \\
\text { Minuten für alle be- } \\
\text { rücksichtigten Be- } \\
\text { triebszustände }\end{array}$ \\
\hline 3 & (Ja) & $\begin{array}{c}\text { Ineffizienter Betrieb } \\
\text { bei erhöhter mecha- } \\
\text { nischer Belastung }\end{array}$ \\
\hline 4 & Nein & $\begin{array}{c}\text { Obere Hysterese- } \\
\text { Schwelle des Ab- } \\
\text { wärtswandlers wird } \\
\text { vielfach nicht über- } \\
\text { schritten }\end{array}$ \\
\hline
\end{tabular}

\section{Einsatz im realen Bahnbetrieb}

Für den Feldtest wurden sechs EnergyHarvesting-Sensoren zur Detektion von Flachstellen in einem Gehäuse an sechs der acht Radlagerdeckel eines Kesselwagens befestigt. Die Anordnung ist in Abb. 12 auf dem oberen Foto dargestellt. Die herausgeführten Kabel dienen der Überwachung der Systeme während des Versuchs. Für den eigentlichen Betrieb sind sie nicht notwendig. Es erfolgten mehrere Messfahrten auf einer Kurzstrecke

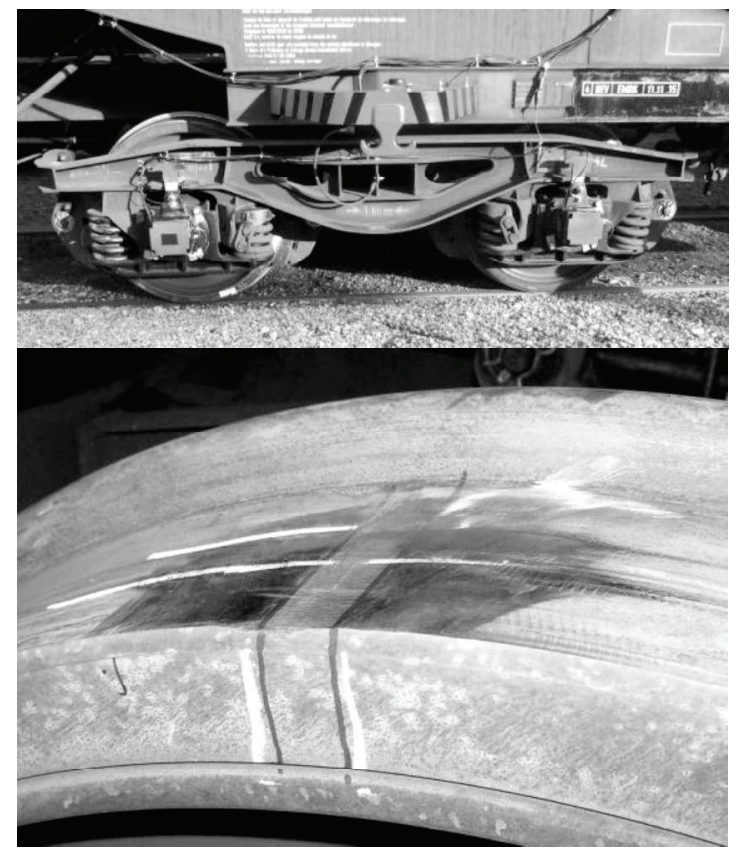

Abb. 12: Testbetrieb von 6 Energy-HarvestingSensoren zur Detektion einer $20 \mathrm{~mm}$ Flachstelle im realen Bahnbetrieb. Zur Versorgung wurden EnergyHarvesting-Generatoren vom Typ 2 (Tab. 2) eingesetzt. von Ludwigshafen nach Kaiserslautern und zurück sowie auf einer Langstrecke von Ludwigshafen nach Schwarzheide und zurück mit beladenem und unbeladenem Kesselwagen im Rahmen des regulären Fahrplans. Für den Test auf der Kurzstrecke wurde eines der Räder mit einer Flachstelle mit einer Länge von $20 \mathrm{~mm}$ geschädigt (Abb. 12, unteres Foto). Diese Flachstelle wurde während der Fahrt von dem an dieser Position montierten EnergyHarvesting-Sensor erkannt. Als Auszug aus den Ergebnissen sind in Abb. 13 die Zuggeschwindigkeit und die entsprechende Ladespannung des Energiespeichers eines EnergyHarvesting-Sensors an einer nicht geschädigten Radposition auf der Teilstrecke Ludwigshafen und Kaiserslautern abgebildet. Es ist zu erkennen, dass genügend Energie generiert wird um alle 10 Minuten die Flachstelle zu detektieren.
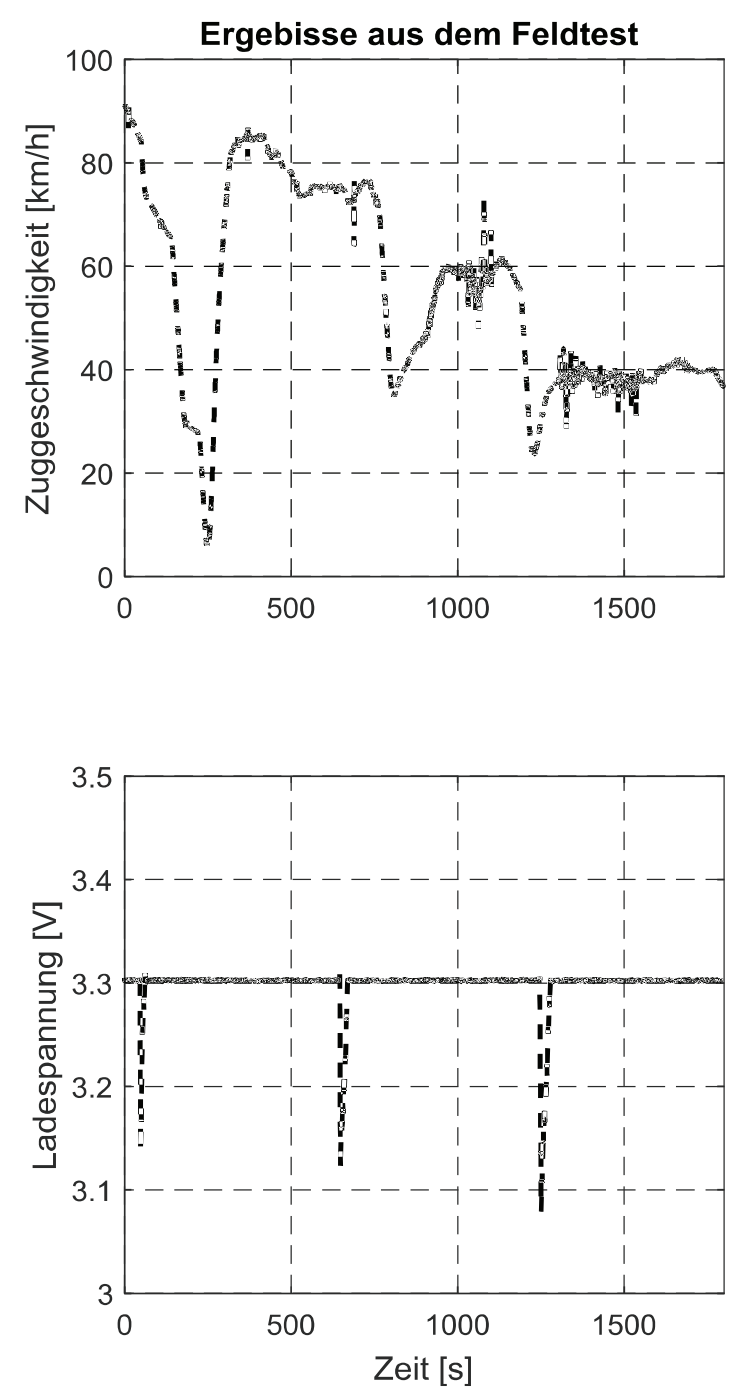

Abb. 13: Auszug aus den Ergebnissen des Feldtests. 


\section{Literaturnachweis}

[1] www.enocean.com (14.03.2016)

[2] A. Kansal, J. Hsu , S. Zahedi, M. B. Srivastava, Power management in energy harvesting sensor networks, ACM Transactions on Embedded Computing Systems (TECS), v. 6 n.4, p.32-es, September 2007

[3] M. Koch, W. Kaal, "Investigation of the operational vibration characteristics of a Freight Car to design Energy Harvesting Sensors", 31th Danubia-Adria Symposium, Kempten University, Germany, 2014.

[4] http://www.lbf.fraunhofer.de/de/projekteprodukte/energieautarkesensorsysteme zustandsueberwachung.html (14.03.2016)

[5] http://www.ts3gmbh.de (14.03.2016)

[6] Liang, J.; Liao, W.-H. Energy flow in piezoelectric energy harvesting systems. Smart Mater. Struct. 2011, 20, 015005.

[7] Guyomar D, Badel A, Lefeuvre E and Richard C 2005 Toward energy harvesting using active materials and conversion improvement by nonlinear processing IEEE Trans. Ultrason. Ferroelectr. Freq. Control 52 584-94

[8] http://www.invent-gmbh.de (14.03.2016)

[9] M. Koch. „Methodische Auslegung energieautarker Systeme". Masterarbeit. Hochschule Koblenz, 2012 